\title{
RESEARCH ON CONSTRUCTING DEM WITH POINT CLOUD FILTERING ALGORITHM CONSIDERING SPECIAL TERRAIN
}

\author{
Chuanli Kang ${ }^{1,2}$, Mingming Zong ${ }^{1,2 *}$, Yao Cheng ${ }^{1,2}$, Fen Wang ${ }^{1,2}$, Tuanneng $\mathrm{Lu}^{1,2}$,Guangzu Liu ${ }^{2}$ \\ ${ }^{1}$ Guangxi Key Laboratory of Spatial Information and Geomatics, Guilin 541004,China \\ ${ }^{2}$ College of Geomatics and Geoinformation, Guilin University of Technology, Guilin 541006, China
}

\section{Commission VI, WG I/2}

KEY WORDS: DEM, Airborne Lidar, Delaunay, Kriging, Filtering algorithm

\begin{abstract}
:
With the development of airborne LiDAR, the use of LiDAR point cloud to construct DEM model is a hot topic in recent years. For the characteristics of time cloud filtering and poor validity, and the efficiency of non-ground point filtering is not high, the filtered point cloud has problems such as errors and leaks. This paper proposes a method of constructing DEM based on the point cloud filtering algorithm of airborne Lidar point cloud data considering special terrain. The experiment proves that the algorithm of this paper is effective for establishing DEM model, and the quality of DEM model is good.
\end{abstract}

\section{INTRODUCTION}

Airborne Lidar Technology (LIDAR) is an active measurement method that can quickly acquire high-precision, high-density three-dimensional spatial information in large-area measurement areas ${ }^{[1]}$. The degree of automation is high, and it is less affected by the weather. It can block through the woods, and the spatial and temporal resolution of the data is relatively high. The digital elevation model contains a wealth of surface and landform feature information, which is a digital description of the ground shape through the ruled discrete grid sampling data. It can simulate the surface undulations well using the limited data with elevation attributes ${ }^{[2]}$. The use of point cloud technology to generate DEM models is a development trend and an important step in point cloud data processing, which plays an indispensable role in the generation and application of subsequent digital products. The amount of point cloud data is highly discrete. How to effectively use the interpolation method to achieve high-precision DEM model has become an urgent problem in the case of reducing manual intervention.

\section{PRINCIPLE}

At present, there are few researches on special terrain. At present, most of the mature algorithms are effective for constructing DEM models in cities and flat areas. However, the accuracy of DEM model generation for both mountains and flat areas is not very good. This paper proposes a point cloud processing method that takes into account special terrain. First, the last echo is filtered out in the original data as the basic point cloud data. The filtered cloud data is grossly eliminated and the wrong data is eliminated. The elevation value of the point cloud is extracted, and the gradient of the adjacent point cloud is calculated by the elevation, and the slope is divided into two types of $0-30,30-60$. The point cloud data is classified according to angles and divided into flat areas and mountains. The point cloud data of each area is separately filtered to extract ground points and non-ground points. Manually rejecting a small number of rough points that have not been filtered makes the modeling accuracy higher. The method of neighbor search of the kd tree is used on the basis of the extracted ground points, so that the searched points form a closed polygon to establish an irregular grid of the area. According to the Delaunay triangulation algorithm, the point-by-point insertion method is used to establish an irregular triangular network TIN, and the TIN is used to approach the ground to make it more in line with the field fluctuation state. Then use the Kriging interpolation method and the inverse distance weighting method to perform elevation interpolation, and finally generate the DEM model.

\subsection{Extract slope}

At present, the accuracy of DEM model construction with both mountains and flat areas is not too good. The complexity of DEM model terrain surface is another factor that must be considered when sampling terrain data. The complexity of the terrain can be described by roughness and irregularities. The terrain surface can be completely formed by the slope.

The first-order differential function of the slope's topographic surface function, expressing the ratio of elevation to distance. The variability of the slope is the second-order differential of the terrain surface, reflecting the complexity of the terrain. There is a strong correlation between the regional DEM elevation accuracy and the average slope value, and the accuracy of the DEM can be predicted by the average slope of the model.The slope expresses the degree of inclination of the terrain unit of the terrain surface (the steep direction and size of the surface) by the elevation change of the two coordinate axes perpendicular to each other. The mathematical model of the

slope is the angle between the normal vector $\mathrm{n}^{\overrightarrow{ }}$ and the $\mathrm{Z}$ axis

of the surface differential unit of the specified point, which reflects the inclination of the specified point, that is, the angle between the tangent plane passing through the designated point of the surface and the horizontal ground. .

Mathematical expression is

$$
\text { Slope }=\operatorname{ArcCos}\left(\frac{\overrightarrow{Z \cdot} \cdot \overrightarrow{\mid}}{|Z| \cdot \mid \overrightarrow{|n|}}\right)
$$


A simplified form of differential publicity:

$$
\text { Slope }=\arctan \sqrt{f_{x}^{2}+f_{y}^{2}} \times 180 / \pi
$$

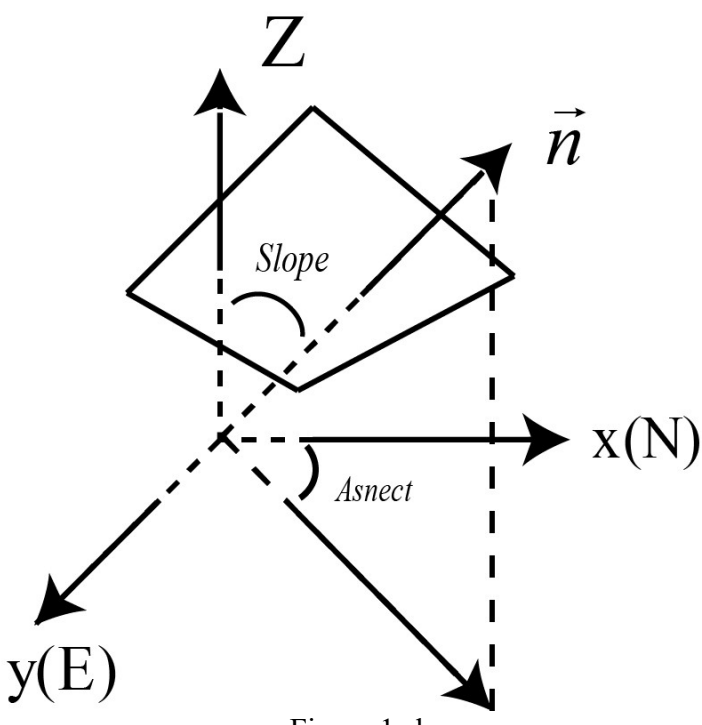

Figure 1 slope

Fx represents the rate of change of the elevation in the $\mathrm{X}$ direction, and fy represents the rate of change of the elevation in the $\mathrm{Y}$ direction. The terrain relief is the difference between the maximum and minimum elevations at a given location, and the terrain relief can visually screen the surface features. The expression is:

$$
\mathrm{R} F_{i}=H_{\max }-H_{\min }
$$

RFi refers to the terrain relief, Hmax is the maximum elevation value in the analysis window, and Hmin refers to the minimum elevation value in the analysis window.

\subsection{KdTree}

KdTree is a data structure that divides the k-dimensional data space. It is mainly used for the search of key data in multidimensional space, which is a special case of binary space segmentation tree. It has great advantages in the neighborhood search. However, in the case of large data volume, if the partition granularity is small, the overhead of building a tree is relatively large, and it will be more flexible than the octree. In the case of small data volume, the search efficiency is compared. high. KdTree is a recursive proximity search strategy that consists of two main aspects: building KdTree and finding it in KdTree. The neighboring point of a point only needs to search in its parent node and child node, which greatly reduces the search scale of the neighboring point. KdTree can effectively judge the insertion point and its nearest point is at that position, which is very important for low-level vision.

\subsection{TIN}

The regular moment shopping net data is equally spaced in the horizontal and vertical directions. The plane coordinates of the grid points are hidden in the row and column numbers, and are often stored in a matrix structure, or the elevation values of each grid unit are recorded one by one. In addition, data structures such as a run length coding structure, a block coding structure, and a quadtree coding structure are used to store DEM data.
An irregular model is created based on the undulating state of the terrain. The data obtained by the regular grid sampling method and the progressive sampling method, especially the square grid data, is most suitable for grid-based surface modeling ${ }^{[3]}$. Usually grid modeling is often used to process authority data covering a flat area. For special terrain, a method of establishing an irregular grid should be used.

Irregular grids are much more complicated than regular grids. Due to the irregularity of triangles, the definition of triangles and their adjacent relationships are to be expressed. The TIN model not only stores the elevation of each vertex, but also Stores the plane coordinates of the vertices of the triangle, the connection relationship between the vertices, and the topological relationship such as the adjacent triangle. Irregular triangulation uses fewer points to return higher precision and has a good topological relationship. Irregular grids are constructed by fitting multiple triangles to discrete data to fit the true ground. It is a good reflection of the undulating shape of the earth's surface, especially in valleys and ridges. The more common method for constructing irregular triangulation is the Delaunay triangle. The method of splitting.

1 , divide and conquer the algorithm, the points are refined according to the region, the triangulation is merged, and the local optimization is performed;

2 , the triangle is first established by the neighboring method, and then the triangulation is established by using the point-bypoint insertion method. Define the minimum outer rectangle range to include as a simple bulge. The search data point $\mathrm{p}$ contains a triangle of points, and $p$ is connected with three vertices of the triangle to form three triangles, and the entire area is optimized from the inside to the outside;

3. The implementation process starts from an arbitrary point and finds the shortest distance point. Connected to the line, according to the method of triangulation, find the point containing another triangle edge, form a new triangulation, and then extend to the entire area.

\subsection{Interpolation}

The inverse distance weighted interpolation algorithm calculates the distance between the coordinates of the point and all the discrete points as the weight influence value. The farther from the sampling point, the smaller the influence of the sampling, that is, the inverse relationship, the reciprocal can have the second constraint. The weakening of the weight is declining. Its formula is:

$$
\begin{gathered}
D_{Z}=\sum_{i=0}^{n} D_{i} * V_{i} \\
D_{i}=\frac{\frac{1}{c+d_{i}^{W}}}{\sum_{i=1}^{n} \frac{1}{C+d_{i}^{W}}}
\end{gathered}
$$

The inverse distance interpolation method is based on the similar principle, that is, the closer the two objects are, the more similar their properties are. Centering on the interpolation point, when the variable $\mathrm{Z}$ value is interpolated at the points $\mathrm{X}$ and $\mathrm{Y}$ to be interpolated, the weighted average of each data point is calculated in the local neighborhood. The formula is:

Where $\mathrm{Z} i$ is the value of the estimated point, $\mathrm{Z} 0$ is the measured value of the interpolated element at the $i$-th point, and 
$\mathrm{n}$ is the number of the measured points participating in the interpolation.

Kriging interpolation is one of the classical algorithms of geostatistics, and it is a method for unbiased optimal estimation of the value of regionalized variables in a finite region.

DEM is a digital set of undulating points in the y-domain and expressing the ground undulating state in elevation. It is a method for continuously expressing spatial fluctuations ${ }^{[4]}$. The DEM model can be better established only when the ground point is accurately extracted.

\section{EXPERIMENT}

Partial Lidar data scanned by Jyväskylä, a city in central Finlan $\mathrm{d}$, acquired by Blom in 2011 .

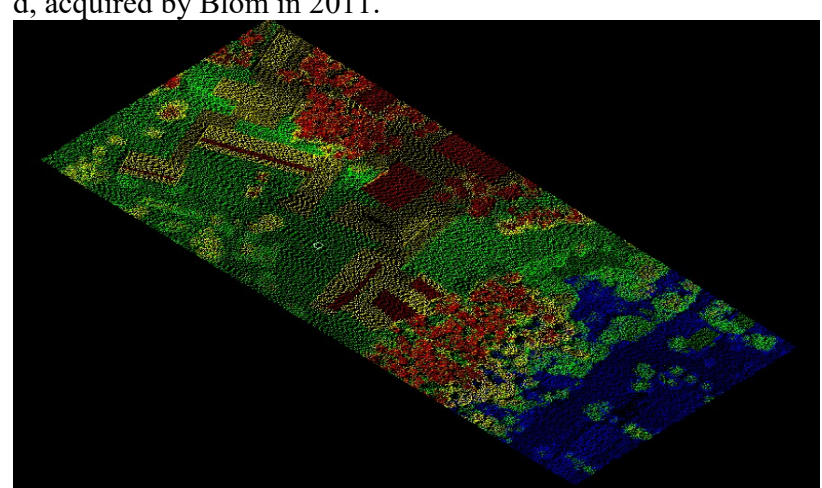

Figure 2 Point cloud data

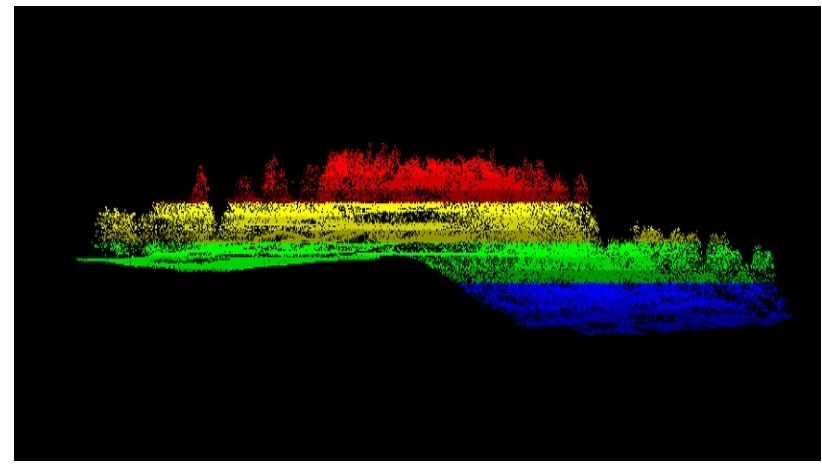

Figure 3Point cloud data

The obtained point cloud data is processed by point cloud data. The point cloud data gross rejection eliminates the data that does not match the required quality and the data that does not belong to the required area to ensure the quality of the point cloud data. The elevation value of the point cloud is extracted, and the gradient of the adjacent point cloud is calculated by the elevation, and the slope is divided into two types of 0-30, 30-60. The point cloud data is classified according to angles and divided into flat areas and mountains. Subsequent processing of the two types of terrain.

The neighbor search performs gross error rejection on the obtained point cloud data, and it is necessary to set a nearsearch neighbor important parameter such as a k-nearest neighbor's sphere radius and a neighbor distance multiplier. The KdTree proximity search needs to set the maximum distance of the connection point to determine the spherical radius setSearchRadius (1.5) of the k-nearest neighbor, the multiplier setMu (2.5) of the neighbor distance, and the maximum number of nearest neighbors setMaximumNearestNeighbors (100). It plays a very important role in establishing a high quality irregular triangulation.

In the process of obtaining data from LiDAR, noise will inevitably occur. Therefore, data filtering is performed to remove the random error factors in the original data to improve the terrain representation accuracy of the DEM of data quality. The main purpose of noise filtering is to separate points below the ground or points that are not in the desired area, and points that are significantly above the ground from the point cloud data. A point or set of points is compared as a seed to a range of points around the perimeter, and points that exceed the threshold are separated from the point cloud data.

Point cloud classification to distinguish between ground and non-ground points. The bare ground point has only one return band, and the position of the echo reflection is the position of the ground point. The ground point of the coverage area is the point at which the last echo of the multiple echoes is reflected ${ }^{[4]}$. The position of the callback point is the elevation corresponding to the feature point. To establish the DEM, it is necessary to extract the accurate ground point. If the accuracy of the ground point extraction is insufficient, the quality of the generated DEM model is poor, and the effect is not ideal.

Manual editing, because of the influence of the algorithm, not all areas can complete the point cloud filtering process, which will generate some faulty areas, resulting in the processed data can not meet the quality requirements of DEM data results, so human-computer interaction is required. It is necessary to manually delete the missing point cloud data, so that the factors affecting the DEM model are minimized.

In this paper, the point cloud data is respectively used by Kriging interpolation method and inverse distance weighting method. The artificially edited data is generated into DEM model, and the experimental comparison is made. The area of 0 $30^{\circ}$ is suitable for using Kriging interpolation method, 30- The $60^{\circ}$ area is suitable for the inverse distance weighting method. The Kriging interpolation method is applicable to flat areas, and the inverse distance weighting method is applicable to mountains. For special terrains, we should use the combination of Kriging and inverse distance weighting methods to establish DEM. The DEM effect of this method is better.

\section{IN CONCLUSION}

In this paper, the process of rapid processing of airborne LiDAR point cloud data to generate DEM is designed, and the theory is used to demonstrate the theory.

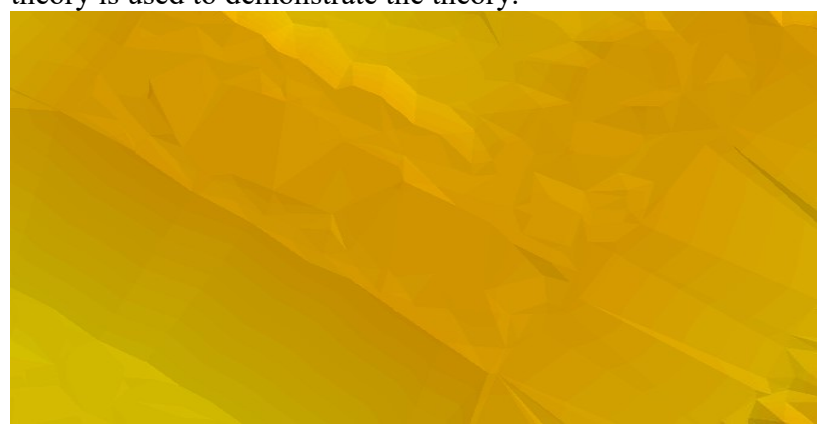

Figure 4 Ordinary model 


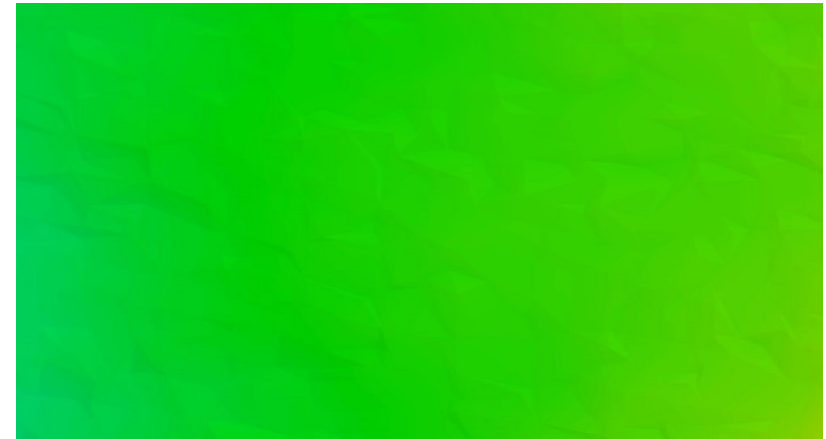

Figure 5 Flat model

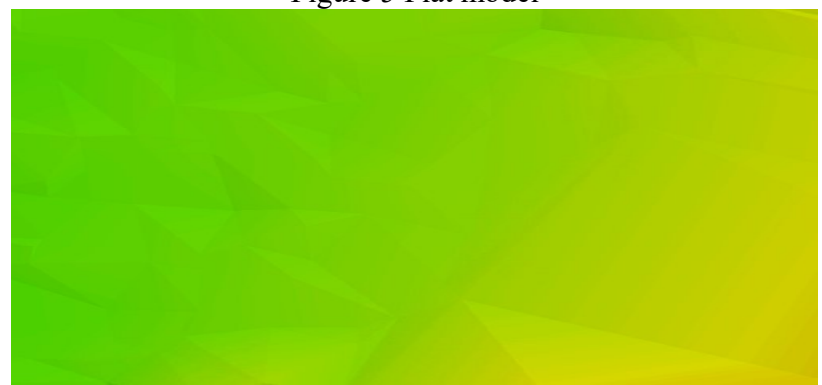

Figure 6 Mountain model

The theory obtained by using the analysis of point cloud data is better in using the Kriging interpolation method in the flat terrain. It is suitable to use the inverse distance weighted interpolation method in the mountains. This method takes into account flat terrain and mountains. This method works better than using a single algorithm. In the experiment, the effect of point cloud classification is not very good and needs further optimization.

\section{ACKNOWLEDGEMENTS}

This paper is financially supported by the Guangxi Innovative Development Grand Grant under the grant number:GuikeAA18118038,GuikeAA18242048;Guilin Science and Technology Research and Development Projectthe grant number: 20170220; the “Ba Gui Scholars" program of The provincial government of Guangxi; and the Guangxi Key Laboratory of Spatial Information and Geomatics nubers 140452410,163802501 .

\section{REFERENCES}

[1]Peng Li.Research on Airborne LiDAR Data Filtering for Steep Area with Dense vegetation[D].Chongqing University,2015.

[2]Xu Jing.Research on Key Technologies of Constructing Fine City Digital Elevation Model[D].Xi'an University of Science and Technology,2015.

[3]Zhang Zhimu, Cai Yinfeng, et al.Comparison of DEM Surface Modeling Based on TIN and Grid.Copper engineering, 2005(02):8-10.

[4]Liu Shanshan.Discussion on Data Processing Method of Point Cloud in DEM Production. Geomatics \& Spatial Information Technology ,2018,41(08):172-174

[5]Li Xiaohong.A Fast Algorithm of Li DAR Point Clouds to Generate Grid DEM.Surveying and mapping bulletin, 2012(12):44-46.

[6]Sithole G , Vosselman G . Experimental comparison of filter algorithms for bare-Earth extraction from airborne laser scanning point clouds[J]. Isprs Journal of Photogrammetry \& Remote Sensing, 2004, 59(1-2):85-101.

[7] Hu H, Ding $Y$, Zhu Q , et al. An adaptive surface filter for airborne laser scanning point clouds by means of regularization and bending energy[J]. ISPRS Journal of Photogrammetry and Remote Sensing, 2014, 92:98-111.

[8]Zhao X, Guo Q , Su Y, et al. Improved progressive TIN densification filtering algorithm for airborne LiDAR data in forested areas[J]. ISPRS Journal of Photogrammetry and Remote Sensing, 2016, 117:79-91.

[9]Podobnikar T, Vre?Ko A. Digital Elevation Model from the Best Results of Different Filtering of a LiDAR Point Cloud[J]. Transactions in GIS, 2012, 16(5):603-617.

[10]Chen C , Li Y, Li W , et al. A multiresolution hierarchical classification algorithm for filtering airborne LiDAR data[J]. ISPRS Journal of Photogrammetry and Remote Sensing, 2013, 82:1-9.

[11]Zeng Z , Wan J , Liu H . An entropy-based filtering approach for airborne laser scanning data[J]. Infrared Physics \& Technology, 2016, 75:87-92.

[12]Shi W, Deng S, Xu W. Extraction of multi-scale landslide morphological features based on local $G i$ * using airborne LiDAR-derived DEM[J]. Geomorphology, 2018, 303:229-242.

[13]Zhan C, Ma H, Liang Z. Model transfer-based filtering for airborne LiDAR data with emphasis on active learning optimization[J]. Remote Sensing Letters, 2018, 9(2):111-120.

[13]Liu L, Lim S. A Voxel-based Multiscale Morphological Airborne Lidar Filtering Algorithm for Digital Elevation Models for Forest Regions[J]. Measurement, 2018, 123:135-144.

[14]Mora $O$, Lenzano $M$, Toth $C$, et al. Landslide Change Detection Based on Multi-Temporal Airborne LiDAR-Derived DEMs[J]. Geosciences, 2018, 8(1):23.

[15]Barišin I, Hinojosa-Corona A, Parsons B. Co-seismic vertical displacements from a single post-seismic lidar DEM: example from the 2010 El Mayor-Cucapah earthquake[J]. Geophysical Journal International, 2018, 202(1):328-346. 